SU-ITP-94-1

January 1994

hep-th/9401070

\title{
Black Hole Entropy in Canonical Quantum Gravity and Superstring Theory
}

\author{
LeONARD SussKind $^{\star}$ AND John UGLUM ${ }^{\dagger}$ \\ Department of Physics \\ Stanford University, Stanford, CA 94305-4060
}

\begin{abstract}
In this paper the entropy of an eternal Schwarzschild black hole is studied in the limit of infinite black hole mass. The problem is addressed from the point of view of both canonical quantum gravity and superstring theory. The entropy per unit area of a free scalar field propagating in a fixed black hole background is shown to be quadratically divergent near the horizon. It is shown that such quantum corrections to the entropy per unit area are equivalent to the quantum corrections to the gravitational coupling. Unlike field theory, superstring theory provides a set of identifiable configurations which give rise to the classical contribution to the entropy per unit area. These configurations can be understood as open superstrings with both ends attached to the horizon. The entropy per unit area is shown to be finite to all orders in superstring perturbation theory. The importance of these conclusions to the resolution of the problem of black hole information loss is reiterated.
\end{abstract}

PACS categories: 04.70.Dy, 04.60.Ds, 11.25.Mj, 97.60.Lf

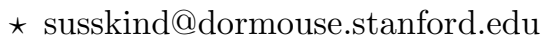

$\dagger$ john@dormouse.stanford.edu 


\section{Introduction}

In conventional statistical mechanics, the entropy of a system originates in the counting of quantum states which are macroscopically indistinguishable. The Bekenstein-Hawking entropy associated with a black hole of mass $M \gg M_{\text {Planck }}$ has never been explained as a consequence of such state counting. In fact, as will be shown, in the theory of quantum fields propagating in a fixed black hole background, the entropy stored in quanta near the horizon is divergent. In other words, an infinite number of macroscopically indistinguishable states of the quantum field exist arbitrarily close to the horizon. This is in obvious contradiction with the finiteness of the Bekenstein-Hawking entropy. This conflict is at the root of the information paradoxes of Hawking. As an example, consider a process in which informationcarrying photons are dropped into a black hole at an average rate which compensates the Hawking evaporation. If one studies this process in the usual approximation of quantum fields in a fixed black hole background, one discovers a contradiction. After a long while, the information stored by photons near the horizon is much larger than that permitted by the Bekenstein-Hawking entropy. Thus, it is claimed that information is lost. The real problem is that there is a conflict between the entropy defined by state counting (which is infinite) and by black hole thermodynamics (which is finite). In this paper it will be shown that the entropy divergences obtained by state counting are closely related to conventional ultraviolet divergences of canonical quantum gravity, and therefore that the information puzzles are part of the problem of the nonrenormalizability of the theory.

On the other hand, string theory is an ultraviolet finite theory of gravity. In an earlier paper, it was speculated by one of us that string theory resolves the puzzles behind the Bekenstein-Hawking entropy, both by producing a finite entropy and providing an explicit counting of states [1]. In this paper we provide strong evidence to support these speculations. We will show that the counting of string states near a horizon gives a finite entropy which agrees with the usual Bekenstein-Hawking result

$$
\sigma_{B H}=\frac{4 \pi M^{2}}{G}=\frac{A}{4 G}
$$

Furthermore, we will be able to identify the string configurations which lead to this result. Thus, in string theory there is no way to hide information in excess of $\sigma_{B H}$ near the horizon. It must be reemitted in the radiation. 
We will begin by considering the entropy of an eternal Schwarzschild black hole using canonical quantum gravity. Using the Euclidean functional integral formulation of the partition function for canonical quantum gravity coupled to matter, we will show that the Bekenstein-Hawking formula (1.1) is a general result, but that the gravitational coupling $G$ appearing in equation (1.1) is the renormalized gravitational coupling. In particular, this means that the divergences in the entropy due to matter fields are the same divergences one must deal with when trying to renormalize the theory. This shows that the question of the finiteness of the entropy is inextricably intertwined with the renormalization of the gravitational coupling, and therefore cannot be understood without a complete knowledge of the ultraviolet behavior of the theory. We then show that in its perturbative formulation, the theory of superstrings propagating in an eternal black hole background gives rise to a completely finite entropy of the form (1.1), because the renormalization of $G$ in superstring theory is finite. We then demonstrate that the entropy arises from counting states of open strings with both ends attached to the horizon.

Even before attempting any calculations, however, a problem arises: to perform a careful statistical mechanical computation for a black hole with a temperature $\tau$, the black hole must be in thermal equilibrium with a radiation bath, and this radiation permeates all of spacetime. Therefore, one should expect a divergence, proportional to the volume of space, in any extensive quantities, such as the Helmholtz free energy. This problem can be avoided by considering the limit of an infinitely massive black hole, for which the Hawking-Unruh temperature is zero. The resulting geometry outside the event horizon is described by the Rindler metric. Beginning with the Schwarzschild metric

$$
g=-\left(1-\frac{2 G M}{r}\right) d t \otimes d t+\left(1-\frac{2 G M}{r}\right)^{-1} d r \otimes d r+r^{2} d \Omega_{2}
$$

define new coordinates $T$ and $s$ by

$$
\begin{aligned}
T & =\frac{t}{4 G M} \\
s & =\sqrt{8 G M(r-2 G M)},
\end{aligned}
$$

so that $g$ may be written

$$
g=-s^{2}\left(1+\frac{s^{2}}{16 G^{2} M^{2}}\right)^{-1} d T \otimes d T+\left(1+\frac{s^{2}}{16 G^{2} M^{2}}\right) d s \otimes d s+4 G^{2} M^{2}\left(1+\frac{s^{2}}{16 G^{2} M^{2}}\right)^{2} d \Omega_{2} .
$$

Taking the limit $M \rightarrow \infty$, the spherical horizon surface becomes planar, and equation (1.4) 
becomes the Rindler metric

$$
g=-s^{2} d T \otimes d T+d s \otimes d s+d x^{2} \otimes d x^{2}+d x^{3} \otimes d x^{3} .
$$

In order to regulate divergences coming from the infinite area of the Rindler horizon, we shall put in an infrared cutoff by defining $x^{2}, x^{3} \in\left[-\frac{L}{2}, \frac{L}{2}\right]$, and then impose suitable boundary conditions on the fields. With this procedure, the area $A$ of the horizon is simply $L^{2}$. The quantities of interest, such as the entropy per unit area, remain well defined in the limit $L \rightarrow \infty$. In addition, the near horizon temperature also remains finite.

Rindler space $\mathcal{R}$ may be viewed as the wedge $x^{1} \geq\left|x^{0}\right|$ of Minkowski space. A Rindler observer at constant $s$ corresponds to a uniformly accelerated observer in Minkowski space. From the point of view of such an observer, Rindler space is causally complete, because the surface $x^{0}=-x^{1}$ corresponds to $T=-\infty$. Signals which pass into Rindler space from the region beneath this surface are viewed as initial data. Now consider a system in the Minkowki space vacuum $|0\rangle_{M}$. A Rindler observer, who can sample only the Rindler wedge, must trace over all degrees of freedom outside Rindler space. He will therefore view the Minkowski vacuum as a mixed state. Remarkably, the density operator for the Rindler observer is [2]

$$
\rho_{R}=\frac{\operatorname{EXP}\left(-2 \pi H_{R}\right)}{Z}
$$

which corresponds to a thermal ensemble at the Rindler temperature $\tau_{R}=\frac{1}{2 \pi}$. The Rindler Hamiltonian $H_{R}$ is given in terms of the Minkowski space energy momentum tensor as

$$
H_{R}=\int_{\mathcal{R}} d^{2} x d s s\left(T_{M}\right)^{00},
$$

where the integral is evaluated on the surface $T=0$. 


\section{Statistical mechanics of a scalar field in Rindler space}

In statistical mechanics, the entropy of a system is defined to be

$$
\sigma=-\operatorname{Tr}(\rho \operatorname{LOG}(\rho))
$$

where $\rho$ is the density operator for which equation (2.1) is maximized, subject to constraints. In the microcanonical ensemble, in which the energy $E$ of the system is held fixed, $\rho$ is given by $1 / N$ times the projection operator for the eigenspace corresponding to $E$, where $N$ is the dimensionality of this eigenspace. The entropy is $\sigma=\log (N)$, the logarithm of the dimension of the allowed space of states, and represents a counting of allowed states. The effect of a more general density operator may be to weight states differently, but the entropy can still be interpreted as the logarithm of an effective dimension of a space of states with nonnegligible probability. For example, a system in thermal equilibrium with a heat reservoir at temperature $\tau$ is described by the density operator

$$
\rho=\frac{\operatorname{EXP}(-\beta H)}{Z}
$$

where $\beta=1 / \tau$ is the inverse temperature and $H$ is the Hamiltonian. The quantity $Z$ is the partition function, and is defined by

$$
Z=\operatorname{Tr}(\operatorname{EXP}(-\beta H))=\exp (-\beta F)
$$

where $F$ is the Helmholtz free energy. It is easily shown that the entropy (2.1) can be obtained from the Helmholtz free energy as

$$
\sigma=\beta^{2} \frac{\partial F}{\partial \beta}
$$

The equation (2.3) for the partition function applies to quantum theories of fields propagating on a fixed Lorentzian spacetime manifold $\mathcal{M}$, described by a metric $g$. As long as $\mathcal{M}$ is stationary, so that thermal equilibrium is a well-defined concept, one can select a time-like Killing vector $\left(\partial / \partial x^{0}\right)$ and calculate the partition function. 
Consider now a scalar field propagating in Rindler space $\mathcal{R}$. The action describing the field is

$$
S[\phi]=-\frac{1}{2} \int_{\mathcal{R}} \epsilon_{g}\left((\nabla \phi)^{2}+m^{2} \phi^{2}\right)
$$

where the metric $g$ is given by equation (1.5) and $\epsilon_{g}=\sqrt{-\operatorname{det}\left(g_{\mu \nu}\right)} d T \wedge d s \wedge d x^{2} \wedge d x^{3}$ is the volume form corresponding to $g$. The field equation is the usual Klein-Gordon equation

$$
\left(\nabla^{2}-m^{2}\right) \phi=\left(-\frac{1}{s^{2}} \frac{\partial^{2}}{\partial T^{2}}+\frac{\partial^{2}}{\partial s^{2}}+\frac{1}{s} \frac{\partial}{\partial s}+\frac{\partial^{2}}{\left(\partial x^{2}\right)^{2}}+\frac{\partial^{2}}{\left(\partial x^{3}\right)^{2}}-m^{2}\right) \phi=0 .
$$

$\phi$ may be expanded as

$$
\phi=U(s) \exp \left(-i \omega T+i \vec{k} \cdot \vec{x}_{\perp}\right)
$$

where $\vec{x}_{\perp}=\left(x^{2}, x^{3}\right)$. Imposing periodic boundary conditions in $x^{2}$ and $x^{3}$ requires that $\vec{k}=\frac{2 \pi}{L}\left(n^{2}, n^{3}\right)$, where $\left(n^{2}, n^{3}\right) \in \mathbb{Z}^{2}$. Defining $\xi=\sqrt{\vec{k}^{2}+m^{2}}$, the equation for $U$ may be written

$$
\left(\frac{d}{d s}\left(s \frac{d}{d s}\right)-\frac{(i \omega)^{2}}{s}\right) U(s)=\xi^{2} s U(s)
$$

which is the modified Bessel equation of order $i \omega$ in eigenvalue form. The most general solution of this equation is

$$
U=A I_{i \omega}(\xi s)+B K_{i \omega}(\xi s)
$$

The eigenfunctions and eigenvalues are determined by imposing boundary conditions. The unboundedness of $I_{i \omega}(x)$ as $x \rightarrow \infty$ requires $A=0$. To regulate the theory, we demand that $U$ vanish at $s=\varepsilon$, where $\varepsilon$ is close to zero. Then the eigenvalues are the solutions of the equation

$$
K_{i \omega}(\xi \varepsilon)=0 .
$$

Note that equation (2.10) should be viewed as an equation for the Rindler frequencies $\omega$. The solutions of this equation can be approximated as follows. Define $x=\log (\xi s)$ and $E=\frac{1}{2} \omega^{2}$, 
so that equation (2.8) can be rewritten as a time independent Schrödinger equation

$$
\left(-\frac{1}{2} \frac{d^{2}}{d x^{2}}+\frac{1}{2} e^{2 x}\right) U(x)=E U
$$

for a particle of mass $m=1$ moving in the potential

$$
V(x)= \begin{cases}\frac{1}{2} e^{2 x}, & \text { if } x>\log (\xi \varepsilon) \\ \infty, & \text { otherwise }\end{cases}
$$

The eigenvalues $E$ can be approximated using the WKB method. The turning points of the classical motion occur when $V=E$, or when $x \in\{\log (\xi \varepsilon), \log (\omega)\}$. The WKB quantization condition is

$$
n \pi=\int_{\log (\xi \varepsilon)}^{\log (\omega)} d x \sqrt{2(E-V(x))} .
$$

This integral can be calculated, and leads to the result

$$
n=\frac{\omega}{2 \pi}\left[\log \left(\frac{1+\sqrt{1-(\xi \varepsilon / \omega)^{2}}}{1-\sqrt{1-(\xi \varepsilon / \omega)^{2}}}\right)-2 \sqrt{1-(\xi \varepsilon / \omega)^{2}}\right]
$$

which is an implicit equation for the frequencies $\omega$. Requiring that the square root be real gives the condition $\omega \geq \xi \varepsilon$. The eigenfrequencies depend on both the quantum number $n$ and the wave vector $\vec{k}$, and are denoted by $\omega_{n}(\vec{k})$.

The partition function for a single mode, labeled by the quantum numbers $n$ and $\vec{k}$, is given by

$$
Z(\beta ; n, \vec{k})=\sum_{m=0}^{\infty} e^{-m \beta \omega_{n}(\vec{k})}=\left(1-e^{-\beta \omega_{n}(\vec{k})}\right)^{-1}
$$

Since the modes are independent, the total partition function is

$$
Z(\beta)=\prod_{n, \vec{k}} Z(\beta ; n, \vec{k})=\exp (-\beta F(\beta))
$$

and the Helmholtz free energy is

$$
F(\beta)=-\frac{1}{\beta} \sum_{n, \vec{k}} \log (Z(\beta ; n, \vec{k}))
$$


Approximating the sums by integrals, equation (2.17) becomes

$$
F(\beta)=\frac{L^{2}}{\beta} \int_{\mathbf{R}^{2}} \frac{d^{2} k}{(2 \pi)^{2}} \int_{\xi \varepsilon}^{\infty} d \omega \frac{d n}{d \omega} \log \left(1-e^{-\beta \omega}\right)
$$

Differentiating equation (2.14) gives the density of levels

$$
\frac{d n}{d \omega}=\frac{1}{2 \pi} \log \left(\frac{1+\sqrt{1-(\xi \varepsilon / \omega)^{2}}}{1-\sqrt{1-(\xi \varepsilon / \omega)^{2}}}\right),
$$

and changing the orders of integration, one obtains the expression

$$
F(\beta)=\frac{A}{(2 \pi)^{2} \beta} \int_{\varepsilon m}^{\infty} d \omega \log \left(1-e^{-\beta \omega}\right) \int_{0}^{\sqrt{(\omega / \varepsilon)^{2}-m^{2}}} d k k \log \left(\frac{1+\sqrt{1-(\varepsilon / \omega)^{2}\left(k^{2}+m^{2}\right)}}{1-\sqrt{1-(\varepsilon / \omega)^{2}\left(k^{2}+m^{2}\right)}}\right),
$$

where $L^{2}=A$ is the area of the horizon. After performing the integral over $k$, equation (2.20) becomes

$F(\beta)=\frac{A}{(2 \pi)^{2} \beta} \int_{\varepsilon m}^{\infty} d \omega \log \left(1-e^{-\beta \omega}\right)\left[(\omega / \varepsilon)^{2} \sqrt{1-(\varepsilon m / \omega)^{2}}+\frac{m^{2}}{2} \log \left(\frac{1-\sqrt{1-(\varepsilon m / \omega)^{2}}}{1+\sqrt{1-(\varepsilon m / \omega)^{2}}}\right)\right]$.

Expanding in powers of the field mass $m$, the leading term is

$$
F(\beta)=\frac{A}{(2 \pi \varepsilon)^{2} \beta} \int_{0}^{\infty} d \omega \omega^{2} \log \left(1-e^{-\beta \omega}\right)
$$

which is integrated to yield

$$
F(\beta)=-\frac{\pi^{2} A}{180 \varepsilon^{2} \beta^{4}} .
$$

The resulting entropy, evaluated at the Rindler temperature $\tau_{R}=1 / 2 \pi$, is

$$
\sigma_{\phi}=\frac{A}{360 \pi \varepsilon^{2}} .
$$

The entropy of the $\phi$ field diverges quadratically with the cutoff $\epsilon$. This divergence is proportional to the area of the horizon, however, and only the numerical coefficient depends 
on the cutoff procedure. The nature of this divergent entropy can be understood in terms of the infinite gravitational redshift between the horizon and infinity. Any field mode with finite frequency at the horizon must have vanishing frequency at infinity. Therefore, the dimension of the space of states with arbitrarily small energy is infinite. The regulator $\varepsilon$ is an ultraviolet regulator, which has the effect of cutting off the sum over states. The result (2.24) agrees with the entropy of a scalar field propagating outside a finite mass black hole as calculated by 't Hooft [3].

Because the thermal density operator in Rindler space can be obtained from the Minkowski vacuum by tracing over the degrees of freedom outside the Rindler wedge, the above calculation is related to the calculation performed by Srednicki [4]. Srednicki calculated the entropy resulting from tracing over the degrees of freedom of a scalar field contained within a spherical cavity when the field is in the Minkowski vacuum state. In the limit of a large sphere, the entropy per unit area calculated in [4] should agree with that in Rindler space. The result (2.24) is in qualitative agreement with the results in [4], although the numerical coefficient of the quadratically divergent entropy is substantially different. This can be attributed to the sensitive dependence of quadratic divergences on the regulator method.

\section{The Bekenstein-Hawking entropy and the renormalization of $G$}

The divergent entropy of the scalar field obtained in Chapter 2 is certainly nonsensical, and an obvious flaw in the calculation is that the backreaction of the gravitational field has been neglected. Since field states with arbitrarily high energy were included in the partition function, one should expect modifications to the background geometry. The argument may be raised, therefore, that a calculation which includes this backreaction may very well lead to a finite entropy. In what follows this is addressed. We will calculate the entropy of an infinitely massive, eternal Schwarzschild black hole by (formally) evaluating the functional integral of Euclidean canonical quantum gravity. We find that the question of the finiteness of the entropy can be answered only by understanding the renormalization of the gravitational coupling $G$.

For completeness, we begin by formulating the functional integral representation of the

partition function. If a spacetime manifold $\mathcal{M}$ is static, there exist coordinates $\left\{x^{\mu}\right\}$ such 
that the metric may be written

$$
g=g_{00} d x^{0} \otimes d x^{0}+g_{i j} d x^{i} \otimes d x^{j}
$$

where $g_{\mu \nu}$ is independent of $x^{0}, i, j \in\{1,2,3\}$, and $\mathcal{M}$ has the topology $\mathbb{R} \times \Sigma$. Next, define the Euclidean manifold $\overline{\mathcal{M}}$, with topology $S^{1} \times \Sigma$, by defining the Euclidean "time" coordinate $\theta=i x^{0}$ and periodically identifying $\theta$ with period $\beta$. The metric on $\overline{\mathcal{M}}$ then has signature +4 , and is written

$$
\bar{g}=-g_{00} d \theta \otimes d \theta+g_{i j} d x^{i} \otimes d x^{j}
$$

The partition function for fields $\phi$ propagating on $\mathcal{M}$ can be expressed as a functional integral

$$
Z(\beta)=\mathcal{N} \int \mathcal{D}[\phi] e^{-I[\phi]},
$$

where $\mathcal{N}$ is a normalization factor and $I$ is the action of the theory on the Euclidean manifold. This method of calculation, while derived from the Hamiltonian formulation of the quantum field theory, has the advantage of being generally covariant. The formalism is extendable to include stationary as well as static spacetimes.

For the theory of canonical quantum gravity, in which the metric is one of the fields to be integrated over, the generalization of equation (3.3) is taken to define the partition function [5]. The partition function is formally written as

$$
Z(\beta)=\mathcal{N} \int_{\mathcal{F}} \mathcal{D}[g] \int \mathcal{D}[\phi] \exp (-I[g, \phi]),
$$

where the space $\mathcal{F}$ of Euclidean metrics is restricted by boundary conditions, such as the total energy contained in spacetime and behavior at infinity. The Euclidean action functional $I$ appearing in the integral is

$$
I[g, \phi]=I_{E H}[g]+I_{\phi}[\phi, g],
$$

where $I_{E H}$ is the Euclidean Einstein-Hilbert action

$$
I_{E H}[g]=\frac{1}{16 \pi G_{0}}\left(-\int_{\overline{\mathcal{M}}} \epsilon_{g} R+2 \int_{\partial \overline{\mathcal{M}}} \epsilon_{h} K\right),
$$

and $I_{\phi}$ is the action of the "matter" (non-gravitational) fields $\phi$. The bare gravitational coupling is explicitly denoted by $G_{0}$. 
The usual method of calculation of the partition function (3.4) is to find a manifold $\overline{\mathcal{M}}$ with metric $\hat{g}$ which is a stationary point of the classical action and satisfies the boundary conditions. Then, writing an arbitrary metric $g$ as $g=\hat{g}+f$, one quantizes the fluctuations $f$ and $\phi$ in the background metric $\hat{g}$. The action (3.6) can be expanded in powers of $f$ as

$$
\begin{aligned}
I[g, \phi] & =I[\hat{g}+f, \phi] \\
& =I_{E H}[\hat{g}]+I_{\phi}[\phi, \hat{g}]+\left.\int_{\frac{\mathcal{M}}{\hat{M}}} \epsilon_{\hat{g}} \frac{\delta I}{\delta g}\right|_{\hat{g}} f+\left.\frac{1}{2} \int_{\frac{\mathcal{M}}{}} \epsilon_{\hat{g}} \frac{\delta^{2} I}{\delta g^{2}}\right|_{\hat{g}} f^{2}+\ldots,
\end{aligned}
$$

and the partition function (3.4) can be written

$$
Z(\beta)=e^{-\beta F}=\exp \left(-I_{E H}[g]\right) Z^{\prime}
$$

where

$$
Z^{\prime}=\mathcal{N} \int \mathcal{D}[f] \int \mathcal{D}[\phi] \exp \left(-\left(I[\hat{g}+f, \phi]-I_{E H}[\hat{g}]\right)\right)
$$

To study the partition function for gravitational and matter fields propagating outside an infinitely massive, eternal black hole, the stationary point to expand around is a Euclidean continuation $\overline{\mathcal{R}}$ of Rindler space, with metric

$$
g=s^{2} d \theta \otimes d \theta+d s \otimes d s+d x^{2} \otimes d x^{2}+d x^{3} \otimes d x^{3}
$$

The Euclidean "time" coordinate $\theta$ is periodic with period $\beta$, and we again restrict $x^{2}, x^{3} \in$ $\left[-\frac{L}{2}, \frac{L}{2}\right]$ to regulate divergences due to the horizon area $A$. Now consider a subspace of constant $x^{2}$ and $x^{3}$. This subspace has the geometry of a cone with angle deficit $(2 \pi-\beta)$, and consequently has a conical singularity at the origin $s=0$ proportional to $(2 \pi-\beta)$. However, $s=0$ corresponds to the event horizon of the black hole, which has no curvature singularity in the Lorentzian geometry. Imposing the condition that this singularity be absent leads to the result that the correct Euclidean continuation has $\beta=2 \pi$. (This is one method of deriving the Hawking-Unruh temperature, because even for a finite mass black hole, the Rindler space approximation becomes arbitrarily good as one gets close to the horizon.) Indeed, only if $\beta=2 \pi$ will the metric be a stationary point of the Euclidean action (3.6). 
Nevertheless, in order to obtain the entropy by means of equation (2.4), one must know the partition function for $\beta$ slightly different from $2 \pi$, so one must consider the geometries containing conical singularities. Indeed, it is shown in [6] that the entropy is conjugate to the deficit angle. A Euclidean continuation of Rindler space with period $\beta$ will be denoted $\overline{\mathcal{R}}_{\beta}$. It would seem that these geometries fail to be stationary points of the functional integral. Note, however, that the derivative with respect to $\beta$ appearing in equation (2.4) is a partial derivative. Thus, all other thermodynamic variables must be held fixed, including the horizon area $A$. The condition that $A$ remain fixed must be implemented in the functional integral. This can be achieved by means of a Lagrange multiplier. The effect of this multiplier is to insert an energy density along the surface $s=0$, which is analogous to a cosmic string. The solution to the classical equations of motion will therefore have a conical singularity at $s=0$. Thus the Euclidean continuations for $\beta \neq 2 \pi$ are stationary points of the Euclidean functional integral subject to the constraint of constant horizon area.

Consider now the factor $\exp \left(-I_{E H}[\hat{g}]\right)$ in equation (3.8), which gives the contribution to the entropy from the classical geometry. The effect of the curvature singularity is that

$$
\int_{\overline{\mathcal{R}}_{\beta}} \epsilon_{\hat{g}} R=2 A(2 \pi-\beta) .
$$

Thus the Einstein-Hilbert action is

$$
S_{E H}[\hat{g}]=-\frac{(2 \pi-\beta) A}{8 \pi G_{0}}=\beta F
$$

From equation (3.12) one obtains the Bekenstein-Hawking formula for the entropy per unit area,

$$
\frac{\sigma}{A}=\frac{1}{4 G_{0}} .
$$

Note, however, that this entropy does not have any obvious origin in the counting of quantum states, since it arises simply from the classical action of the conical Rindler background.

To proceed, we must (formally) calculate the functional integral $Z^{\prime}$ in equation (3.8). The actual quantization of the theory defined by equation (3.8) is a tricky subject, but we shall find that we do not need more than the most basic of results. First note from equation (3.7) that, neglecting the fluctuations $f, Z^{\prime}$ reduces to the partition function for 
matter fields propagating in the Rindler background. For the case of a single free scalar field, this was calculated in Chapter 2 . From the point of view of the Euclidean functional integral, the computation in Chapter 2 is equivalent to considering the free matter field on a fixed Rindler background $\overline{\mathcal{R}}_{\beta}$ with conical deficit $2 \pi-\beta$. The functional integral can be represented in terms of first quantized particle paths, according to a standard prescription. In higher orders of perturbation theory, the paths branch to form Feynman diagrams. However, the only diagrams which can contribute to the entropy are those which intersect or encircle the horizon at $s=0$. To see why, consider first the lowest order diagrams, which are simple closed curves. A single loop with fixed "center of mass" which does not intersect or encircle $s=0$ is insensitive to the deficit angle. Summing over all such loops leads to an integration of the center of mass of each loop over all angular positions, and the result is proportional to $\beta$. Accordingly, it will give no contribution to the entropy. A loop which encircles the singularity represents a real particle propagating outside the black hole, and this state certainly contributes positively to the entropy. The divergence in equation (2.24) is evidently due to very small loops which encircle $s=0$. It is for this reason that the entropy is localized near the horizon. The point is that calculating the partition function by evaluating the Euclidean functional integral on a conical background provides a unified way of obtaining the entropy due to both the classical geometry and the quantum corrections. However, thus far only the quantum corrections have a clear interpretation in terms of the counting of states.

Integrating out all the matter fields and tree level gravitons gives a contribution proportional to $\frac{1}{\varepsilon^{2}}$. Including this term, the entropy per unit area is

$$
\frac{\sigma}{A}=\frac{1}{4}\left(\frac{1}{G_{0}}+\frac{C}{90 \pi \varepsilon^{2}}\right)
$$

where $C$ is a constant which depends on the matter content of the theory. As we shall see in what follows, the same quadratic divergence enters into the renormalization of the gravitational coupling in the effective action.

We now proceed to prove this statement. After integrating out the matter fields and fluctuations of the metric, $Z^{\prime}$ has the form $Z^{\prime}=\exp \left(-W^{\prime}\right)$, where on general grounds $W^{\prime}$ must be a diffeomorphism-invariant functional of the background metric $g^{\star} . W^{\prime}$ will contain

\footnotetext{
* Here and henceforth we drop the caret over the background metric $g$.
} 
all possible covariant terms, and may be expanded in powers of the Riemann tensor and its derivatives as

$$
W^{\prime}[g]=\int_{\frac{\mathcal{M}}{\mathcal{M}}} \epsilon_{g}\left[-\frac{1}{16 \pi} a R+Q(R)\right] .
$$

Here $a$ is a constant and $Q$ contains all other induced covariant terms. We neglect a possible renormalization of the cosmological constant. The effect of $a$ is to renormalize the value of the gravitational coupling in the effective action from $G_{0}$ to $G_{R}$, given by

$$
\frac{1}{G_{R}}=\frac{1}{G_{0}}+a
$$

The next step is to evaluate equation (3.15) for Euclidean Rindler space $\overline{\mathcal{R}}$. To regulate the curvature singularity at the origin, define $R=(2 \pi-\beta) f$, where $f$ is a smooth function supported only on an $\varepsilon$-neighborhood of the origin. The condition that (3.11) be satisfied means that $f$ must satisfy

$$
\int_{\overline{\mathcal{R}}_{\beta}} \epsilon_{g} f=2 A .
$$

We also require that the scale of variation of $f$ is independent of the conical angle, so that derivatives of $f$ do not introduce additional dependence on $\beta$. Now consider the possible types of terms that can appear in $Q$.

1. Any local or nonlocal term with $n \geq 2$ powers of the Riemann tensor $R_{\alpha \beta \mu \nu}$ will be proportional to $(2 \pi-\beta)^{n}$. This includes terms with arbitrary numbers of derivative operators acting on $R_{\alpha \beta \mu \nu}$. Their contribution to $W^{\prime}$ may be represented as

$$
-\sum_{n=2}^{\infty} b_{n}(2 \pi-\beta)^{n}
$$

where the $b_{n}$ are constants.

2. Now consider terms linear in $R_{\alpha \beta \mu \nu}$, with arbitrary derivatives acting on them. For example, consider

$$
I=\int_{\overline{\mathcal{R}}} \epsilon_{g}\left(\nabla^{2}\right) R
$$

Since $R$ is now a smooth function, by use of Stokes' theorem equation (3.19) can be rewritten 
as an integral over the boundary of $\overline{\mathcal{R}}$,

$$
I=\int_{\partial \overline{\mathcal{R}}} \epsilon_{h} n^{\mu} \nabla_{\mu} R
$$

where $n$ is a unit vector normal to $\partial \overline{\mathcal{R}}$. But $R$ vanishes outside a small neighborhood of the origin, so the integral $I$ vanishes. It is obvious that all such terms will vanish after integration by parts.

Due to the rapid falloff of the Green functions in four dimensions, nonlocal terms proportional to one power of $R_{\mu \nu \alpha \beta}$ will not appear, and the above list covers all possible terms. Thus, using the condition (3.11), the full Helmholtz free energy $\beta F=S_{E H}[g]+W^{\prime}[g]$ can be written

$$
\beta F=-\sum_{n=1}^{\infty} b_{n}(2 \pi-\beta)^{n}
$$

where $b_{1}=\frac{A}{8 \pi G_{R}}$ comes only from the Einstein-Hilbert term. The entropy is therefore

$$
\sigma(\beta)=\frac{\beta A}{8 \pi G_{R}}+\sum_{n=1}^{\infty}\left(b_{n}+\beta(n+1) b_{n+1}\right)(2 \pi-\beta)^{n}
$$

Setting $\beta=2 \pi$, equation (3.22) reduces to the Bekenstein-Hawking entropy (1.1), but with the renormalized gravitational coupling $G_{R}$ given by equation (3.16).

Thus we arrive at the conclusion that for the case of canonical quantum gravity coupled to matter fields, the expression (1.1) for the Bekenstein-Hawking entropy of the fields propagating outside a black hole is a general result, but the gravitational coupling appearing in equation (1.1) is the renormalized gravitational coupling $G_{R}$ given by equation (3.16). Comparing equations (3.16) and (3.14), we see that the divergences in the entropy are the same divergences which renormalize the gravitational coupling. In particular, this means that the question of the finiteness of the entropy of the black hole is inextricably intertwined with the renormalization of the theory. Canonical quantum gravity is nonrenormalizable, and it is often the case that the only consistent quantum field theory that can be obtained from a nonrenormalizable theory is a free field theory. If this is the case with canonical quantum gravity, then $G_{R}=0$, and the entropy diverges. 


\section{Two dimensional models}

Because of the large amount of attention that has recently been focused on two dimensional toy models of black holes, it is of interest to examine how quantum corrections affect the entropy of a two dimensional black hole. In the following it will be shown that in the two dimensional model proposed by Callan, Giddings, Harvey, and Strominger (CGHS) [7], the divergence in the entropy of scalar fields moving in a black hole background is not the same as the divergence which renormalizes the gravitational coupling. Instead, it provides an infinite zero point entropy, which corresponds to an infinitely degenerate ground state and probably a theory which loses information.

The CGHS model is defined by the action functional

$$
S_{C G H S}=\frac{1}{2 \pi} \int \epsilon_{g}\left(e^{-2 \phi}\left[R+4(\nabla \phi)^{2}+4 \lambda^{2}\right]-\frac{1}{2}(\nabla f)^{2}\right)
$$

where $g, \phi$, and $f$ are the metric, dilaton, and matter fields, respectively, and $\lambda^{2}$ is a cosmological constant which defines a length scale for the theory. The classical theory defined by the action (4.1) has eternal black hole solutions. Defining light cone coordinates $x^{ \pm}$and choosing the line element to have the form $d s^{2}=-e^{2 \rho} d x^{+} d x^{-}$, these solutions are given by

$$
e^{-2 \rho}=e^{-2 \phi}=\frac{M}{\lambda}-\lambda^{2} x^{+} x^{-}
$$

where $M$ is the black hole mass. The future horizon is the curve $x^{-}=0$.

The CGHS theory can be viewed as an effective action for radial modes of near extreme, magnetically charged black holes in four dimensional dilaton gravity [7]. Using this correspondence, the area of the black hole is defined to be $\lambda^{-2} e^{-2 \phi}$ evaluated at the horizon, or $A=M \lambda^{-3}$.

As for any non extreme black hole, the behavior of the geometry near the horizon is closely approximated by Rindler space. Taking the limit $M \rightarrow \infty$, a calculation of the entropy of the scalar field $f$ may be performed using techniques analogous to those used in Chapter 2. Due to the bad infrared behavior of scalar fields in two dimensions, in addition to the horizon cutoff $\varepsilon$ one must also introduce an infrared cutoff $\ell$. The entropy is found to 
be

$$
\sigma=\frac{1}{6} \log \left(\frac{\ell}{\varepsilon}\right)
$$

Note that this entropy is not proportional to the horizon area. Instead, it represents an infinite additive constant to the entropy. Indeed, it can be seen that the entropy of the $f$ field cannot be proportional to the area because of the way $f$ couples to the dilaton. From the point of view of the four dimensional theory, the result (4.3) occurs because the truncation of all but the spherically symmetric modes is a violent reduction of the number of degrees of freedom of the theory.

The origin of this entropy can also be understood by examining the effective action obtained after integrating out the $f$ field. This action is given by the original action (4.1) plus a Liouville action, which can be written using the above metric as

$$
\begin{aligned}
S_{L} & =-\frac{1}{12 \pi} \int d^{2} x(\rho-\log (\ell / \varepsilon))(\nabla)^{2}(\rho-\log (\ell / \varepsilon)) \\
& =-\frac{1}{96 \pi} \int \epsilon_{g} R \frac{1}{\nabla^{2}} R-\frac{\log (\ell / \varepsilon)}{12 \pi} \int \epsilon_{g} R .
\end{aligned}
$$

Note that the $\rho$ field only appears in the combination $\rho-\log (\ell / \varepsilon)$. The first term in equation (4.4) is the familiar correction to the classical action, and is responsible for the Hawking radiation from the two dimensional black hole. The second term is proportional to the Euler class, and is the term which gives rise to the divergent entropy of the scalar field. Indeed, being careful with factors of $\pi$ which enter into definitions of energy in the theory, the entropy (4.3) can be read off from the second term in equation (4.4). It should be pointed out that the first term in the action (4.1) gives rise to the classical contribution to the entropy, and as with the four dimensional black hole, there is no obvious counting of states associated with this entropy.

Since the integrated curvature is a topological invariant, the second term in the effective action (4.4) plays no part in the dynamics of the theory, and is usually ignored. Moreover, its contribution to the entropy is as an additive constant, which has no thermodynamic significance. However, the presence of this term does have information theoretic significance. Due to this infinite additive constant, there is no mechanism to prevent the black hole from accumulating an arbitrarily large amount of information near the horizon. It is therefore highly plausible that information is, in fact, lost in two dimensional theories, or that black 
hole remnants exist in these theories, which amounts to the same thing. Nevertheless, we have seen that a truncation to only spherically symmetric modes does great violence to a four dimensional theory - the entire renormalization structure is profoundly changed. For this reason, we conclude that two dimensional theories do not possess enough degrees of freedom to be viable models of four dimensional gravity.

\section{Superstrings and black hole entropy}

Having learned in Chapter 3 that the question of black hole entropy is related to the ultraviolet divergence structure of our theory of quantum gravity, the next step is to look for theories for which this divergence structure can be understood. A natural candidate for examination is the theory of superstrings propagating in a background spacetime.

There are two essential points we will establish in the remainder of this paper. The first point is that in string theory, unlike canonical quantum gravity, the entire entropy per unit area of a horizon can be attributed to identifiable quantum states. In other words, superstring theory is an "induced" theory of the gravitational effective action, in which all the terms in the action arise from integrations over fluctuations in the presence of a background geometry. This is true even for the "classical" or tree graph action, which is generated by integration over genus zero surfaces. Computing these fluctuations in a conical background requires a definition of the string theory "off-shell". Such off shell continuations introduce ambiguities and divergences into the world sheet sigma model. It will be shown, however, that the entropy is entirely independent of these ambiguities.

The second important point to establish is that string theory is also ultraviolet finite, and therefore leads to a finite entropy per unit area. This follows from the fact that the renormalization of the string coupling constant $\kappa$ (in units of the string tension) is finite order by order in perturbation theory.

In the present state of development of string theory, it is not possible to begin with a Hamiltonian, solve for the eigenvalues, and compute the partition function using equation (2.3). Therefore, we must make an ansatz that the definition (3.3) of the partition function holds in string theory, by which we mean that the logarithm of the partition function is given in terms of a sum of string Feynman graphs in the appropriate Euclidean continuation of the spacetime manifold. This ansatz is supported by results in [8]. 
Using this ansatz, we will show how superstring theory resolves the puzzles behind the Bekenstein-Hawking entropy. We show that the contribution to the entropy per unit area from genus zero string graphs is the leading order "classical" term in the Bekenstein-Hawking formula. This classical term thus has a manifest origin in the counting of quantum states. Next we find that the Bekenstein-Hawking result holds to all orders in superstring perturbation theory, and that the renormalized gravitational coupling appearing in the formula is finite.

The starting point for our discussion is the two-dimensional supersymmetric sigma model describing the propagation of superstrings in a background spacetime metric $g$. The generating functional for the two-dimensional superconformal field theory on a world sheet of genus $n$ is

$$
Z^{(n)}=\frac{\kappa_{0}^{2(n-1)}}{\operatorname{Vol}(\mathcal{G})} \int \mathcal{D}[e] \int \mathcal{D}[\chi] \int \mathcal{D}[X] \int \mathcal{D}[\Psi] \exp (-I[X, \Psi ; e, \chi])
$$

where $X^{\mu}$ and $\Psi$ are the bosonic and fermionic coordinates of the superstring, respectively, $e$ is the world sheet zweibein, and $\chi$ is the gravitino. $\kappa_{0}$ is the bare string coupling, and $\mathcal{G}$ denotes the symmetry group of the two dimensional action $I$, which includes diffeomorphisms, superconformal transformations, and an on shell local supersymmetry. The first step is to gauge fix the world sheet zweibein to $e=e^{\Lambda} \hat{e}$ and the gravitino to $\chi=\rho \lambda$, where $\hat{e}$ is a fiducial zweibein, $\rho$ are the two dimensional Dirac matrices, and $\lambda$ is a Grassmann variable. This introduces reparametrization ghosts $b, c, \beta$, and $\gamma$, and equation (5.1) becomes

$$
Z^{(n)}=\kappa_{0}^{2(n-1)} \frac{\int \mathcal{D}[\Lambda, \lambda]}{\operatorname{Vol}(\mathrm{SC})} \int_{F_{n}} d^{2 m_{n}} \tau \frac{\int \mathcal{D}[X] \mathcal{D}[\Psi] \mathcal{D}[b, c, \beta, \gamma]}{\operatorname{Vol}(\Omega)} \exp \left(-I\left[X, b, c, \beta, \gamma ; e^{\Lambda} \hat{e}, \rho \lambda\right]\right)
$$

Here $\mathrm{SC}$ denotes the group of superconformal transformations, $F_{n}$ is a fundamental region for the integration over the $2 m_{n}$ supermoduli $\tau$, and $\Omega$ denotes the additional subgroup of symmetries which remains after the gauge fixing. $\Omega$ is generated by the conformal Killing vectors and spinors. We imagine regulating the two dimensional field theory by replacing the world sheet by a finite lattice. The volume of the group $\Omega$ is then also naturally regulated $[9,10]$.

In order to cancel the integrals over $\Lambda$ and $\lambda$ against $\operatorname{Vol}(\mathrm{SC})$, the theory must actually be superconformally invariant. For strings propagating in flat $D$ dimensional Euclidean space 
with no background fields, the condition for superconformal invariance is that the dimension take the value $D=10$. For a general supersymmetric sigma model, the conditions for superconformal invariance are more complicated, and in addition to the condition $D=$ 10, the spacetime fields must satisfy the equations of motion of a spacetime action. The superconformal invariance conditions imply the vanishing of the beta functions for the two dimensional quantum field theory, so the theory is ultraviolet finite.

To study the statistical mechanics of superstrings near massive, eternal black holes, we shall be interested in calculating the $Z^{(n)}$ for superstrings propagating in Euclidean Rindler space. In order to calculate the entropy, however, a small conical singularity must be introduced, and the resulting space violates the conditions for superconformal invariance of the theory. Therefore, a prescription must be followed for calculating the off shell generating functionals, and we will continue to use (5.2) as our definition of $Z^{(n)}$. It will be found, however, that the entropy is independent of the prescription used.

Consider first the case of genus zero. After integrating over the world sheet fields and dividing out the volume of $\Omega$, equation (5.2) for $Z^{(0)}$ takes the form

$$
Z^{(0)}=\kappa_{0}^{-2} \frac{\int \mathcal{D}[\Lambda, \lambda]}{\operatorname{Vol}(\mathrm{SC})} F(g ; \varepsilon, \Lambda, \lambda)
$$

where $F$ is a generally covariant functional of the background metric $g$, and also depends on the world sheet regulator parameter $\varepsilon$ and the superconformal parameters $\Lambda$ and $\lambda$. The basic structure of $F$ can be determined by quite general arguments. To begin with, for a fixed value of the regulator, $F$ may be expanded as a sum of integrals of powers of the Riemann tensor and its derivatives. To see why, we return to our regulation of the string theory in which the world sheet is replaced by a finite lattice of points. Then the bosonic part of the functional integral becomes a product of ordinary coupled Gaussian integrals. Because of the exponential damping, the integrand tends to zero quickly when the integration variables are distantly separated. In particular, the image of the worldsheet in the target spacetime will have an extent of order $\ell^{2} \sim \log \left(\frac{1}{\varepsilon}\right)$. Thus, for a fixed value of the regulator, there is no way to introduce any nonlocal behavior with extent larger than $\mathcal{O}(\ell)$ into the generating functional, and a series expansion of the type described above will be possible. It should also be pointed out that the quantity $Z^{(0)}$ is not the effective action for string theory as defined by Tseytlin in [10], but is simply related to it, as is demonstrated in Appendix A. 
The coefficients of the terms in the expansion of $F$ will depend on $\varepsilon, \Lambda$, and $\lambda$, and will in general diverge as $\varepsilon$ goes to zero. This is one of the difficulties involved in defining string theory off shell. It is shown in Appendix A, however, that the coefficient of the term $\int \epsilon_{g} R$ is independent of $\varepsilon, \Lambda$, and $\lambda$. For this term the integral over the superconformal parameters simply cancels $\mathrm{Vol}(\mathrm{SC})$, and so $Z^{(0)}$ can be written

$$
Z^{(0)}=-\kappa_{0}^{-2} \int \epsilon_{g} R+\kappa_{0}^{-2} \frac{\int \mathcal{D}[\Lambda, \lambda]}{\operatorname{Vol}(\mathrm{SC})} Q(g ; \varepsilon, \Lambda, \lambda)
$$

where $Q$ contains all the other terms in $F$.

Although it is apparent that a unique definition of the off shell amplitude does not exist, it is obvious that the first term in equation (5.4) governs the low energy scattering of gravitons, and that its coefficient can be related in the usual way to the bare gravitational coupling.

The genus zero generating functional $Z^{(0)}$ has been written down for a ten dimensional background metric, but we want to study four dimensional physics, so we must introduce a compactification scheme. For simplicity, we will consider a target space $\overline{\mathcal{T}}$ which is a product manifold $\overline{\mathcal{M}} \times K$, where $\overline{\mathcal{M}}$ is a four dimensional manifold coordinatized by $\left\{x^{i}\right\}_{i=1}^{4}$ (which will eventually be identified with four dimensional Euclidean Rindler space), and $K$ is a $D-4$ dimensional compact manifold coordinatized by $\left\{x^{i}\right\}_{i=5}^{D}$ and having no intrinsic curvature. The metric on $\overline{\mathcal{T}}$ is block diagonal, decomposing into a metric $g^{(4)}$ on $\overline{\mathcal{M}}$ and a metric $g^{(D-4)}$ on $K$. A simple choice for $K$ is the product manifold $\left(S^{1}\right)^{D-4}$, with $x^{i} \in\left[0, L_{i}\right]$ for $i \in\{5, \ldots, D\}$, and $g_{i j}^{(D-4)}=\delta_{i j}$. The $L_{i}$ are taken to be on the order of $\sqrt{\alpha^{\prime}}$. The genus zero generating functional can now be written

$$
Z^{(0)}=-\frac{1}{16 \pi G_{0}}\left(\int \epsilon_{g^{(4)}} R+\frac{\int \mathcal{D}[\Lambda, \lambda]}{\operatorname{Vol}(\mathrm{SC})} \frac{\int}{\mathcal{M}} \epsilon_{g^{(4)}} Q\left(g^{(4)} ; \varepsilon, \Lambda, \lambda\right)\right)
$$

where the bare four dimensional gravitational coupling is

$$
G_{0}=\frac{\kappa_{0}^{2}}{16 \pi \int_{K} \epsilon_{g^{(D-4)}}}
$$

All that is left now is to specify the four dimensional manifold $\overline{\mathcal{M}}$ and the background metric. For our purposes, the manifold $\overline{\mathcal{M}}$ is taken to be Euclidean Rindler space $\overline{\mathcal{R}}_{\beta}$, 
which has an angle deficit $(2 \pi-\beta)$, and metric $g^{(4)}$ given by equation (3.10). From here the argument proceeds exactly as in section 3, where we found that the entropy per unit area at $\beta=2 \pi$ depended only on the coefficient of the integral of $R$, and we obtain the result that the entropy per unit area obtained from genus zero string graphs is given by the Bekenstein-Hawking formula,

$$
\frac{\sigma}{A}=\frac{1}{4 G_{0}}
$$

It should by now be apparent that this result does not depend on the exact definition of off shell superstring generating functionals, because changes in the prescription for off shell functionals can only influence the result through the terms which depend on the regulator or the superconformal parameters. These terms all give contributions to the entropy which vanish when one sets $\beta=2 \pi$.

Assuming that superstring theory can be written in a Hamiltonian formulation, it is surprising that the entropy we have obtained is well defined only for the Rindler temperature $\tau_{R}=\frac{1}{2 \pi}$. For ordinary systems, one can calculate $\operatorname{Tr}(\operatorname{EXP}(-\beta H))$ for any value of $\beta$. A similar situation occurs, however, when one tries to calculate the thermodynamics of a sufficiently large gravitating system. Here one is foiled by the fact that the long range gravitational field leads to the Jeans instability, and the thermal ensemble is not well defined. To see the connection with the above calculation, notice that the world sheet ultraviolet regulator $\varepsilon$ acts as a spacetime infrared regulator. For example, a string graph will have an extent of order $\sqrt{\log \left(\frac{1}{\varepsilon}\right)}$, as mentioned previously. The above results suggest that an infrared instability also occurs in our calculation, and that the regulator $\varepsilon$ controls it, allowing one to perform statistical mechanical calculations at an arbitrary temperature. The thermodynamics of the system, and in particular the entropy, will therefore depend on this infrared regulator. The entropy can be made well defined by taking away the regulator only for $\beta=2 \pi$. 


\section{String states on the horizon and the finiteness of the entropy}

The important thing to note about the result (5.7) is that in superstring theory the leading order, "classical" contribution to the Bekenstein-Hawking entropy arises explicitly from an integration over string configurations, namely, those described by genus zero string graphs. It is precisely because of its origin in the counting of string states that the entropy is independent of the exact off shell prescription used - the entropy is an "on shell quantity". The nature of the states contributing to the entropy (5.7) will now be determined.

The bosonic part of a general genus zero superstring graph is a continuous map from $S^{2}$ into the target space $\overline{\mathcal{R}}_{\beta} \times K$, as shown in Figures 1 and 2 . Just as in the case of the first quantized particle paths described in Chapter 3, not all such graphs can contribute to the entropy. Only the graphs which intersect the conical singularity at $s=0$ can contribute. This can be understood as follows. Consider deleting the subspace $s=0$, so that the resulting Euclidean manifold has topology $S^{1} \times \mathbb{R}^{3} \times K$. Since $S^{2}$ is simply connected and the mapping is continuous, the action of a graph cannot depend on $\beta$. Summing over all string graphs involves an integration over the angular location of a given graph, and thus will be proportional to $\beta$. This $\beta$ dependence will be cancelled when one obtains the Helmholtz free energy from the generating functional. On differentiating $F$ with respect to $\beta$, the sum vanishes.

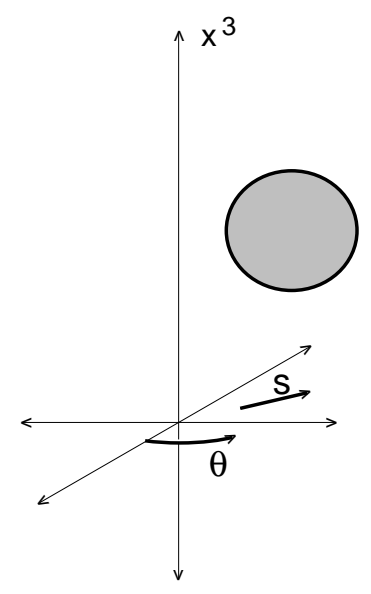

FIGURE 1. A genus zero string graph which does not contribute to the entropy.

All other directions besides $s, \theta$, and $x^{3}$ are suppressed. 


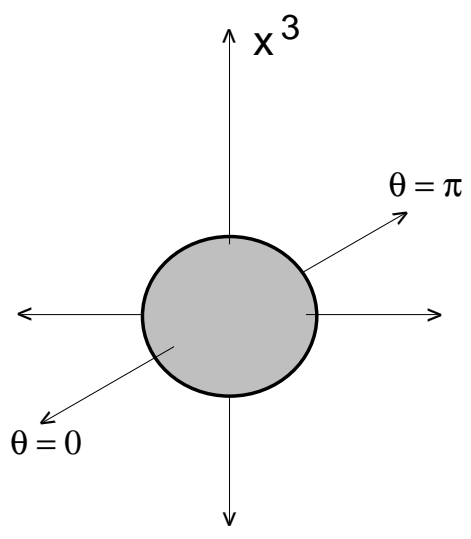

FIGURE 2. A genus zero string graph which does contribute to the entropy.

An example of a genus zero graph which does contribute to the entropy is given in Figure 2. To understand what state this corresponds to, we consider a slice of constant Euclidean time, shown in Figure 3. It is apparent that the states we are counting are closed superstrings which lie partially behind the horizon. An observer outside the black hole describes these states as open superstrings with both ends frozen to the horizon, but which are free to interact with each other and with superstrings propagating outside the black hole. Thus we have proved our first point, that the classical contribution to the Bekenstein-Hawking entropy arises from counting identifiable states of superstrings frozen to the horizon.

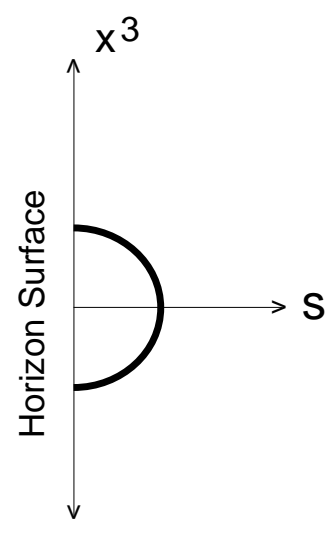

FIGURE 3. A slice of constant Euclidean time showing an open string with both ends attached to the horizon.

Now consider contributions from higher genus graphs. After performing the functional 
integrals over the superstring coordinates and the ghosts, each $Z^{(n)}$ can be expressed in a form similar to equation (5.5). Using the now familiar arguments of Chapters 3 and 5 , the only terms we are interested in are the integrals of the scalar curvature, which are superconformally invariant, so we drop the rest. Denote the genus $n$ contribution as

$$
J^{(n)}=-a_{n} \int \epsilon_{g^{(4)}} R
$$

The effect of the coefficients $a_{n}$ is to renormalize the bare gravitational coupling to its renormalized value $G_{R}$. It is well known that the integrals over the world sheet moduli are the analogues of the integrals over Feynman-Schwinger parameters in quantum field theory. In field theory, these integrals lead to divergences, such as those which lead to infinities in the entropy. It is also well known that in superstring theory the dangerous regions of moduli space are eliminated, and the coefficients $a_{n}$ are finite [11]. Thus the renormalized coupling constant is finite. We therefore arrive at our second conclusion, that the entropy per unit area of a horizon is finite to all orders in superstring perturbation theory, and is given by

$$
\frac{\sigma}{A}=\frac{1}{4 G_{R}}
$$

where $G_{R}$ is the finite renormalized value of the four dimensional gravitational coupling.

As an aside, for certain superstring theories the renormalization of the gravitational coupling is in fact zero. This is the case when, after compactifying the theory down to four dimensions, the theory exhibits an $N=4$ supersymmetry. If the supersymmetry is broken to some lower $N$, then the renormalization of the gravitational coupling is finite but nonzero $[12]$.

As with the genus zero case, only certain higher genus graphs contribute to the entropy. By an argument similar to the above, one can easily see that the only graphs which contribute to the entropy must encircle or intersect the conical singularity at $s=0$. Examples of such genus one graphs are given in Figures 4 and 5. Figure 4 depicts a closed superstring which remains outside the black hole for all time. Such graphs represent states in which the entire string remains outside the horizon. Figure 5 describes a process in which an open string emits and reabsorbs a closed string.

Now an interesting puzzle arises. Consider the fact that in $N=4, D=4$ compactifications of superstring theory (as well as ordinary $N=4, D=4$ supergravity theories) 
the higher loop corrections to the gravitational coupling vanish. By our result in section 5 (section 3), this implies that the corrections to the entropy vanish as well. The fact that the gravitational coupling is not renormalized can be attributed to a cancellation between fermionic and bosonic degrees of freedom in Feynman graphs. However, if one performs an ordinary statistical mechanical calculation of the entropy that counts physical states, then these states, whether bosonic or fermionic, always contribute positively to the entropy. The question is, from the point of view of the statistical mechanical calculation, where do the necessary negative contributions to the entropy come from?

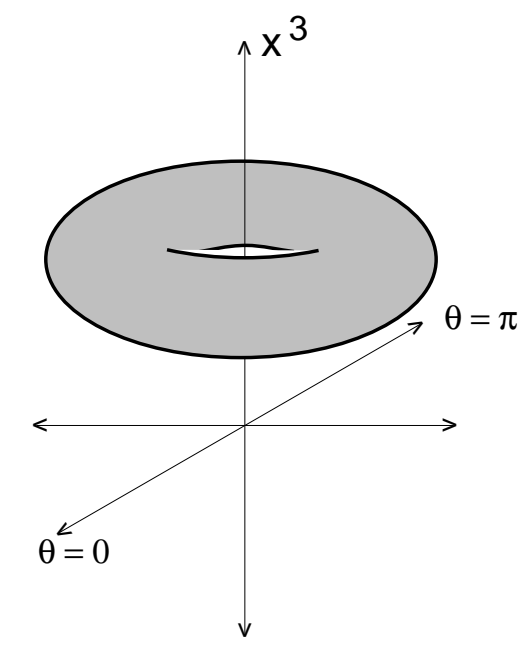

FIGURE 4. A genus one string graph which contributes positively to the entropy.

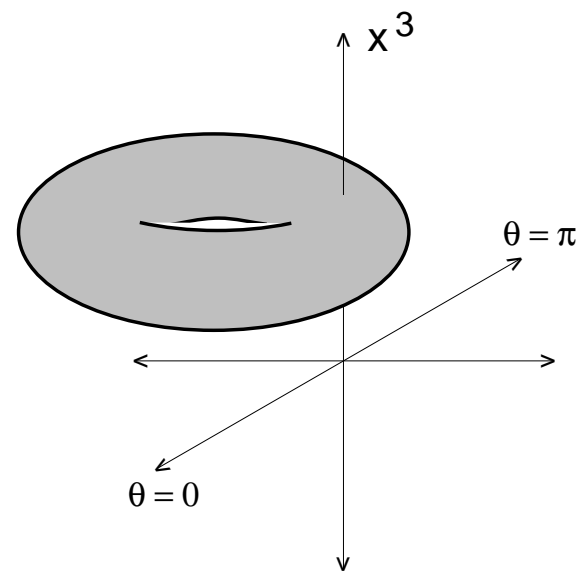

FIGURE 5. A genus one string graph describing a coupling between strings on the horizon and strings outside the horizon. 
In superstring theory, the resolution of this puzzle comes from genus one graphs which intersect the horizon as in Figure 5. These graphs do not represent the contribution of either open strings attached to the horizon, or closed strings outside the horizon. They describe processes which couple the open and closed string sectors. In other words, these graphs describe an interaction between the degrees of freedom outside the horizon and those that make up the horizon. These graphs do not have to contribute positively to the entropy, and in fact must provide the negative contributions to the entropy which cancel the positive contributions from the closed string graphs shown in Figure 4.

\section{Conclusions}

Let us now take stock of what has been learned. In Chapter 2 it was shown that quantum fields propagating in a fixed four dimensional Rindler space background have an entropy per unit area which diverges near the horizon. This divergence is due to an infinitely large number of states having arbitrarily small energy. In Chapter 3 it was shown that this divergence is the same divergence which renormalizes the gravitational coupling in the effective action of canonical quantum gravity. For this reason, the divergence in the entropy cannot be understood without an understanding of the ultraviolet behavior of the theory. In Chapter 4, two dimensional toy models of black holes were examined, and it was found that the matter fields in the theory contribute an infinite additive constant to the entropy. It is therefore reasonable to expect that these models do in fact exhibit information loss. It was argued, however, that these models do not possess enough degrees of freedom to be good models of four dimensional black holes.

In the last two chapters we showed how superstring theory, in its perturbative formulation, resolves the puzzles of the Bekenstein-Hawking entropy. The leading order classical contribution to the entropy per unit area was shown to arise from superstrings which lie

partially behind the horizon, and which act like open strings with both ends attached to the horizon. This result gives a new physical picture of a black hole - that of a surface covered with bits of string which are free to interact with each other and strings outside the black hole. These bits of string give rise to a microstructure on the horizon, which can be thought of in the field theory limit as an additional set of degrees of freedom, which one could call a stretched horizon [13]. 
In addition, the entropy per unit area has been shown to be finite to all orders in superstring perturbation theory, and to be given by the Bekenstein-Hawking formula, equation (6.2). This is because the renormalized gravitational coupling is finite. Therefore, in superstring theory, a black hole cannot absorb an infinite amount of information, and must emit the information in the form of Hawking radiation.

The results obtained in this paper only apply in the limit of infinite black hole mass. For large but finite black hole mass $M$, one would in general expect corrections to the black hole entropy which are of $\mathcal{O}(1 / M)$ compared to the Bekenstein-Hawking formula. We do not doubt the existence of these terms, and it is our belief that these terms will be finite in superstring theory. Nevertheless, given a sufficiently large black hole, Hawking's information problem can be formulated without mention of these terms, so the resolution of the question of information loss should depend only on the finiteness of the Bekenstein-Hawking term.

It should be emphasized that the validity of our result rests on the ansatz for the logarithm of the partition function used in Chapter 5. A true calculation of the entropy would require an actual enumeration of states, for which a Hamiltonian is needed. In the absence of a Hamiltonian, however, the calculation above appears to be the most viable alternative. If superstring theory is truly consistent, it is reasonable to expect that the Hamiltonian calculation will reproduce the results obtained here.

\section{APPENDIX}

In this appendix we show that the coefficient of the term $\int \epsilon_{g} R$ in $F$ is independent of $\varepsilon, \Lambda$, and $\lambda$. We will use results obtained by Tseytlin in reference [10]. Define the quantity $\tilde{Z}^{(0)}$ using equation (5.2) by

$$
Z^{(0)}=\kappa_{0}^{-2} \frac{\int \mathcal{D}[\Lambda, \lambda]}{\operatorname{Vol}(\mathrm{SC})} \frac{1}{\operatorname{Vol}(\Omega)} \tilde{Z}^{(0)}
$$

so that $\tilde{Z}^{(0)}$ is the regulated generating functional for the two dimensional field theory, computed in a particular conformal gauge, and without the volume of $\Omega$ removed from it. It is shown in [10] that $\operatorname{Vol}(\Omega) \propto \log (\varepsilon)$, and that there exists a field redefinition such that

$$
\frac{\partial \tilde{Z}^{(0)}}{\partial(\log (\varepsilon))}=I_{e f f}
$$

where $I_{\text {eff }}$ is the spacetime action which generates equations of motion equivalent to the 
superconformal invariance conditions. $I_{e f f}$ has an expansion of the form

$$
I_{e f f}=c \int \epsilon_{g}\left(-R+\frac{4}{(D-2)}(\nabla \Phi)^{2}+\frac{1}{3} \exp \left(\frac{8 \Phi}{D-2}\right) H^{2}+\alpha^{\prime} Q\left(g ; \alpha^{\prime}, \varepsilon, \Lambda, \lambda\right)\right)
$$

where $c$ is a constant and $Q$ contains terms which are higher order in $R_{\alpha \beta \mu \nu}$, with coefficients that depend on $\alpha^{\prime}, \varepsilon, \Lambda$, and $\lambda$. We can now integrate equation (A.3) with respect to $\log (\varepsilon)$ to obtain $\tilde{Z}^{(0)}$, and insert this quantity in equation (A.1). Dividing out the volume $\operatorname{Vol}(\Omega) \propto \log (\varepsilon)$, we see that the coefficient of the integral of $R$ is independent of $\varepsilon, \Lambda$, and $\lambda$. For this term, the integral over the superconformal parameters cancels $\operatorname{Vol}(\mathrm{SC})$, and we arrive at equation (5.4).

Acknowledgements:

We would like to thank S. Shenker and R. Kallosh for helpful discussions. This work is supported in part by National Science Foundation grant PHY89-17438. J. U. is supported in part by a National Science Foundation Graduate Fellowship.

\section{REFERENCES}

1. L. Susskind, Some Speculations About Black Hole Entropy in String Theory, Rutgers University preprint RU-93-44, August 1993, hep-th/9309145.

2. W. G. Unruh, Phys. Rev. D14 (1976), 870.

3. G. 't Hooft, Nucl. Phys. B256 (1985), 727.

4. M. Srednicki, Phys. Rev. Lett. 71 (1993), 666.

5. G. W. Gibbons and S. W. Hawking, Phys. Rev. D15 (1977), 2752.

6. M. Bañados, C. Teitelboim, and J. Zanelli, Phys. Rev. Lett. 72 (1994), 957;

S. Carlip and C. Teitelboim, The Off Shell Black Hole, preprint IASSNS-HEP-93-84, November 1993, gr-qc/9312002.

7. C. G. Callan, S. B. Giddings, J. A. Harvey, and A. Strominger, Phys. Rev. D45 (1992), 1005.

8. J. Polchinski, Commun. Math. Phys. 104 (1986), 37.

9. J. Liu and J. Polchinski, Phys. Lett. B203 (1988), 39. 
10. A. A. Tseytlin, Phys. Lett. B208 (1988), 221.

11. M. B. Green, J. H. Schwarz, and E. Witten, Superstring Theory, Vol. 2, Cambridge University Press, 1987.

12. V. Kaplunovsky, private communication.

13. L. Susskind, L. Thorlacius, and J. Uglum, Phys. Rev. D48 (1993), 3743. 